\title{
Whole-Body CT in Multiple Trauma Patients: Clinically Adapted Usage of Differently Weighted CT Protocols
}

\section{Polytrauma-Ganzkörper-CT: Klinisch adaptierter Einsatz unterschiedlich gewichteter CT-Untersuchungsprotokolle}

Authors

Stefan Ulrich Reske ${ }^{1,2}$, Rainer Braunschweig ${ }^{1,5 *}$, Andreas Wolfgang Reske ${ }^{3}$, Reinhard Loose ${ }^{4}$, Michael Wucherer ${ }^{4}$

Affiliations

1 Department of Diagnostic Imaging and Interventional Radiology, BG-Klinikum Bergmannstrost Halle, Halle (Saale), Germany

2 Department of Diagnostic and Interventional Radiology and Neuroradiology, Heinrich-Braun-Klinikum, Zwickau, Germany

3 Department of Anaesthesiology, Intensive Care, Emergency Medicine and Pain Therapy, Heinrich-BraunKlinikum, Zwickau, Germany

4 Institute of Medical Physics, Nuremberg General Hospital, Paracelsus Medical University, Nuremberg, Germany

5 Steinhorst, Germany

Key words

multiple trauma, whole-body CT, protocol optimization, arm positioning, dose reduction, clinically adapted approach

received 24.12.2017

accepted 07.05.2018

Bibliography

DOI https://doi.org/10.1055/a-0643-4553

Published online: 12.11.2018

Fortschr Röntgenstr 2018; 190: 1141-1151

(c) Georg Thieme Verlag KG, Stuttgart · New York

ISSN 1438-9029

Correspondence

Stefan Ulrich Reske

Institut für Diagnostische und Interventionelle Radiologie und Neuroradiologie, Heinrich-Braun-Klinikum gemeinnützige GmbH, Karl-Keil-Straße 35, 08060 Zwickau, Germany

Tel.: ++49/3 75/51554838

stefanreske@gmail.com

\section{ABSTRACT}

Purpose Whole-body CT ( $\mathrm{WbCT}$ ) has been established as an internationally accepted diagnostic modality in multiple trauma. Until 2011, a uniform CT scanning protocol was used for all multiple trauma patients (pat.) at our hospital (OLD protocol = OP). In 2011, 2 new differently weighted protocols

* Board Member, Working Group Imaging Procedures of the Musculosceletal System of the German Röntgen Society, Berlin, Germany. were introduced: TIME protocol (TP) for hemodynamically unstable pat. and DOSE protocol (DP) for pat. with stable vital parameters. The aim of this study was to compare the original "One-fits-all-concept" with the new, clinically oriented approach to wbCT.

Materials and Methods This study retrospectively evaluated 3 distinct wbCT protocols, looking at automatic exposure control variation (AEC; OP/TP) and arm positioning close to the body/overhead (TP/DP). The analysis included waist circumference (WC, cm), injury severity score (ISS), examination time (ET, min), image noise (IN), and effective dose (E, mSv). Normality of distribution was assessed with the KolmogorovSmirnov test. Data are given as median and range. Test of significance with Kruskal-Wallis test or Mann-Whitney-U-test. Level of significance: 0.05 .

Results 308 pat. were included in the study $(77 \% \mathrm{~m}$; age: $46 \mathrm{a}, 18-90 \mathrm{a}$; WC: $93 \mathrm{~cm}, 66-145 \mathrm{~cm})$. ISS was 14 (OP; $n=104 ; 0-75), 18$ (TP; $n=102 ; 0-75)$ and 9 (DP; $\mathrm{n}=102 ; 0-50)$. ET was $3.9 \mathrm{~min}(\mathrm{OP} ; 3.3-5.6 \mathrm{~min}), 4.1 \mathrm{~min}$ (TP; $2.8-7.2 \mathrm{~min}$ ) and $7.7 \mathrm{~min}$ (DP; $6-10 \mathrm{~min})$. IN showed no significant differences when comparing OP/TP but was significantly reduced in DP. For a wbCT (vertex to ischium), E could be reduced from $49.7 \mathrm{mSv}$ to $35.4 \mathrm{mSv}$ by optimizing AEC (OP/TP). Through the overhead repositioning of the arms in DP, a further reduction to $28.2 \mathrm{mSv}$ was achieved.

Conclusion AEC and arm repositioning have a crucial influence on image quality and dose. The presented clinical approach is superior to the original concept.

\section{Key Points:}

- The use of 2 differently weighted wbCT protocols allows a more flexible approach to the patient's clinical presentation.

- The clinically adapted concept presented in this study allows trauma care centers to reduce the collective dose.

- Whole-body CT is leading to exposure to relevant radiation doses - further multicenter research is required.

\section{Citation Format}

- Reske SU, Braunschweig R, Reske AW et al. Whole-Body CT in Multiple Trauma Patients: Clinically Adapted Usage of Differently Weighted CT Protocols. Fortschr Röntgenstr 2018; 190: 1141-1151 


\section{ZUSAMMENFASSUNG}

Ziel Die Ganzkörper-CT (GKCT) ist als Primärdiagnostik in der Versorgung von schwerverletzten Patienten (Pat.) weltweit etabliert. Bis 2011 wurden alle Pat. in unserer Klinik mit dem ALT-Protokoll (AP) untersucht. Seit 2011 werden 2 different gewichtete Protokolle vorgehalten und adaptiert an den klinischen Zustand des Pat. eingesetzt. Bei instabilen Pat. wird das ZEIT-Protokoll (ZP) und bei kreislaufstabilen Pat. das DOSISProtokoll (DP) verwendet. Ziel dieser Studie war der Vergleich des alten „One-fits-all-Konzepts“ mit dem neuen, klinisch adaptierten Konzept.

Material und Methoden Diese retrospektive Studie evaluiert 3 in Einzelaspekten differente GKCT-Protokolle: Differente Einstellung der Dosismodulation (DM, AP/ZP) und Armlagerung am Körper/über Kopf (ZP/DP). Evaluiert werden Bauchumfang (BU, cm), Injury-Severity-Score (ISS), Untersuchungszeit (UZ, min), Bildrauschen (BR) und effektive Dosis (E, mSv). Test auf Normalverteilung mittels Kolmogorov-Smirnov-Test. Angabe der Ergebnisse als Median und Spannweite.
Signifikanzprüfung mittels Kruskal-Wallis- oder Mann-Whitney-U-Test. Signifikanzniveau: 0,05.

Ergebnisse 308 Pat. erfüllten die Einschlusskriterien (77\% m; Alter: 46 a (18-90a); BU: $93 \mathrm{~cm}(66-145 \mathrm{~cm}))$. Der ISS lag bei 14 (AP; $n=104 ; 0-75), 18$ (ZP; $n=102 ; 0-75)$ bzw. 9 Punkten (DP; $\mathrm{n}=102 ; 0-50)$. Die UZ betrug 3,9 min (AP; 3,3-5,6 min), 4,1 min (ZP; 2,8-7,2 min) bzw. 7,7 min (DP; 6-10 min). Das BR zeigte im Vergleich AP/ZP keine signifikanten Unterschiede, lag beim DP jedoch signifikant niedriger. Für eine GKCT von Scheitel bis Sitzbein konnte im Vergleich AP/ZP durch Optimierung der DM E von 49,7 auf 35,4 mSv reduziert werden. Im Vergleich ZP/DP konnte durch die Armumlagerung über Kopf E auf 28,2 mSv weiter reduziert werden.

Schlussfolgerung DM und Armlagerung haben entscheidenden Einfluss auf Dosis und Bildqualität. Das vorgestellte, klinisch adaptierte Konzept ist dem ursprünglichen „One-fitsall-Konzept“ überlegen.

\section{Introduction}

Accidents and injuries are the most common cause of death and permanent disability among Germans under the age of 45 [1]. Optimal care of trauma patients plays a significant socioeconomic role. Efficient emergency room care in the acute phase has a major effect on the survival and long-term prognosis of patients with multiple injuries. Exact knowledge of the pattern of injuries is essential for treating in a targeted manner with defined priorities. Computed tomography (CT) has optimal characteristics for trauma diagnosis due to the short examination time and high sensitivity and specificity both for injuries as well as for trauma-independent, treatment-relevant incidental findings. Whole-body CT ( $w b C T)$ is considered as an independent predictor for the survival of trauma patients and has therefore become internationally established as the diagnostic method of choice [2-4]. Consequently, a rapid increase in the number of whole-body CT examinations in trauma patients has been observed in the last decade (in $201679 \%$ of 33374 patients entered in the TraumaRegister $\mathrm{DGU}^{\circledR}$ underwent whole-body examination) $[5,6]$.

The main point of criticism regarding $\mathrm{wbCT}$ is the relevant radiation exposure [7-9]. Particularly in young patients, there is an increased stochastic risk of developing a radiation-induced malignancy [10]. This is to be offset against the risk of death or permanent damage as a result of the trauma [11]. Therefore, indication determination plays an important role in the selection of the diagnostic concept (wbCT vs. diagnostic imaging performed in steps including ultrasound, $\mathrm{X}$-ray and targeted $\mathrm{CT}$ ). The main problem regarding indication determination is the high heterogeneity of the patient population in relation to the mechanism of trauma, pattern of injuries and clinical presentation. Young patients in particular can temporarily compensate for their circulatory situation despite serious injury thus masking the actual severity of the injuries prior to sudden worsening of their condi- tion. To prevent the risk of undertriage (underestimation of the injury severity) with possibly fatal consequences for the patient or medicolegal consequences resulting from mistreatment, the indication for $\mathrm{wbCT}$ is often determined quickly at many hospitals in the case of a corresponding mechanism of trauma. However, experience in the daily radiology routine has shown that only some patients examined with wbCT meet the definition of polytrauma according to Tscherne ("injuries to multiple regions of the body suffered at the same time with at least one or the combination of the individual injuries being life-threatening"). If the inclusion criteria of the TraumaRegister DGU ${ }^{\circledR}$ (admission of a trauma patient to the emergency room and further treatment at an intensive care unit or intermediate care unit; emergency room admission with exitus letalis prior to admission to the intensive care unit; secondary transfer of a trauma patient with TraumaRegister DGU ${ }^{\circledR}$ documentation having been started at the hospital providing primary care) are taken into consideration, it can be concluded that the number of patients examined with wbCT and not entered in the TraumaRegister DGU ${ }^{\circledR}$ is significantly greater than the number stated above [12].

There are various approaches to indication determination in the literature: Some authors use the white paper or $\mathrm{S3}$ guideline criteria for admitting a patient to the emergency room ( $\triangleright$ Table 1) while others developed special score systems [1315]. To date, there is no final interdisciplinary consensus. This is also shown in particular by the extensive discussion in the study by Gupta regarding trauma surgery and emergency room doctors [16]. There is a risk of an increased rate of wbCT without detection of injury in the case of generalized use of wbCT based solely on the mechanism of trauma. Therefore, almost $51 \%$ of patients in the Gupta study did not have any injury that could be detected on $\mathrm{CT}$ and the injury severity score (ISS) was above 15 points in only $20 \%$ of cases [16]. According to the definition of overtriage 
- Table 1 Criteria for emergency room activation.

\section{grade of recommendation $\mathrm{A}$}

unstable vital parameters

- systolic blood pressure less than

$90 \mathrm{mmHg}$ (age-adapted in children)

- GCS less than 9

- respiratory problems/need for intubation apparent injuries

- penetrating injuries/gunshot wounds of the body/neck region

- fractures of $>2$ proximal long bones

- unstable thorax

- pelvic fractures

- amputation injury proximal to hands/feet

- spinal cord injury

- open cranial injury

- burns covering $>20 \%$ of the body surface and degree $\geq 2 b$ grade of recommendation $B$

type of accident

- fall from a height of greater than $3 \mathrm{~m}$

- traffic accident with:

- front collision with intrusion of $>50-75 \mathrm{~cm}$

- change in speed of delta $>30 \mathrm{~km} / \mathrm{h}$

- pedestrian/bicycle collision

- death of a vehicle occupant

- ejection of a vehicle occupant

Summary taken from the $\mathrm{S} 3$ guideline "Polytrauma/Schwerverletzten-Behandlung". If at least one of these criteria is fulfilled, the trauma patient should be treated in the pre-warned emergency room of a trauma care hospital. The grades of recommendation A, B and 0 correspond to the level of evidence present during the development of the guideline and take the form of "shall", "should" and "can" recommendations, respectively [13]. Many hospitals use these criteria to decide whether or not to perform a whole-body CT scan.

by Wurmb (ISS $\geq 16$ = polytrauma; ISS < 16 = overtriaged for $\mathrm{WbCT}$ ), the overtriage rate was $80 \%$ [17].

In many hospitals one single wbCT protocol is used for all trauma patients. This "one-fits-all” concept was abandoned in our hospital in 2011. Instead two differently weighted protocols are used adapted to the patient's clinical condition. The goal of the present study is to evaluate three different $w b C T$ protocols in relation to examination time, image quality, and dose. The original "one-fitsall” concept is compared to the new clinically adapted concept.

\section{Materials and Methods}

The present retrospective, monocentric and anonymized study was approved by the responsible ethics committee.

All presented data were acquired in the period from 6/1/2010 to $6 / 1 / 2013$ in the clinical routine of a tertiary care hospital for trauma management with the status of a Level 1 trauma center. There are 2 emergency rooms for treating seriously ill/injured patients with one of the two CT units at our hospital being installed directly in one of the emergency rooms. Patient care is performed directly at the CT table, with the patient being positioned in the feet-first position for spatial-logistic reasons. The patient is repositioned by the emergency room team from the transportation means used by emergency medical services to a CT-compatible spine board. wbCT performed early after the primary survey has been the established primary diagnostic method at our hospital since 2003. Emergency room admission of trauma patients - and also determination of the indication for wbCT - were performed according to the criteria of the S3 guidelines ( $\vee$ Table 1 ) [13].

The inclusion and exclusion criteria and the wbCT scans examined in the study period are shown in - Fig. 1. 308 evaluable datasets were included in the study. All evaluated wbCT scans were acquired on the $\mathrm{CT}$ unit installed in the emergency room (Toshiba Aquilion $32^{\circledR}$, software version V3.20GR011, Toshiba Medical Systems Europe, Zoetermeer, The Netherlands). Compliance tests and regular consistency checks were performed according to $\S 16$ of the X-Ray Ordinance. Iterative reconstructions were not available in the study period.

Three different wbCT protocols were evaluated in the study period. The protocol referred to in the following as the "OLD protocol" was used to 2011 for all trauma patients (group O). With the goal of dose optimization, various changes were made in 2011 and two new differently weighted protocols were created ("TIME protocol" - group T and "DOSE protocol" - group D). These were presented to the hospital's own trauma committee and have been used since $8 / 1 / 2011$. The decision as to which of the two new protocols was to be used was made as a function of the patient's clinical condition based on the decision algorithm shown in $>$ Fig. 2.

The same scan segments were used for all three protocols: one native head/neck scan and one contrast-enhanced body scan. In approximately $1 / 3$ of all patients, a leg scan was additionally performed. However, this is not included in the present publication. While the body and leg scans were planned with an overlap in group $\mathrm{O}$, this overlapping was avoided in the new protocols $(T+D)(\triangleright$ Fig. 3$)$.

All head/neck scans were examined without dose modulation (= automatic exposure control $=\mathrm{AEC}$ ) and without gantry tilting. All three protocols used the existing AEC (SUREExposure3D ${ }^{\mathrm{TM}}$, Toshiba Medical Systems Europe, Zoetermeer, The Netherlands) in the body scan. The exact technical specifications of the three protocols are shown in > Fig. 4.

The arms were positioned close to the body in group $\mathrm{O}$ and the complete wbCT scan was planned based on a long whole-body scout. After simultaneous start of the scanner and contrast agent via injector, the wbCT scan was performed automatically: After acquisition of the native head/neck scan, an interscan delay and a venous contrast-enhanced body scan were performed.

Group T used the same automatic examination procedure with the arms close to the body. However, on the basis of a new refer- 


\begin{tabular}{|c|c|}
\hline total number of examined polytrauma wbCT examinations & 645 \\
\hline excluded data sets & 337 \\
\hline underage patients (under 18 years old) & 21 \\
\hline wbCT in transferred patients & 34 \\
\hline wbCT on second CT unit not in the emergency room & 13 \\
\hline missing dose report in the PACS (due to database migration lasting multiple months) & 162 \\
\hline use of other wbCT protocols ( 22 were examined with the DOSE protocol with 1 arm overhead and 1 arm close to the body) & 47 \\
\hline interruption in the examination procedure in the case of emesis & 2 \\
\hline $\begin{array}{l}\text { technical problems while performing the examination (in more than } 75 \% \text { of these wbCT scans, one of the scouts was acquired in only } 1 \text { plane } \\
\text { and not in } 2 \text { planes as planned) }\end{array}$ & 18 \\
\hline $\begin{array}{l}\text { relevant deviations from the previously defined scan segments (particularly shortening of the body scan with exclusion of the neck or } \\
\text { lengthening of the body scan to include the legs) }\end{array}$ & 40 \\
\hline included data sets & 308 \\
\hline OLD protocol (0) & 104 \\
\hline TIME protocol (T) & 102 \\
\hline DOSE protocol (D) & 102 \\
\hline
\end{tabular}

- Fig. 1 This figure shows the number of whole-body CT scans performed in the study period and assessed by inclusion and exclusion criteria. Furthermore, the number of included and excluded scans is given. Included in the study were all primary care patients who underwent whole-body $\mathrm{CT}$ after severe trauma on our emergency room scanner using one of the established protocols. The following data sets were excluded: underage patients, missing dose report in the PACS system, discrepancy between used and established CT protocol, technical problems occurring during the scan and relevant deviation from the primarily defined scan length of the scan regions ( $\bullet$ Fig. $\mathbf{3}$ ).

ence convolution kernel (FC17), the body scan was acquired with a revised AEC setting ( $\bullet$ Fig. 4 ).

Group D was examined with a divided protocol design: After acquisition of a short scout, the native head/neck scan was planned and acquired. The patient's arms were then positioned overhead. The body scan was then planned and acquired on the basis of a new long scout. This additional scout acquisition after arm repositioning is absolutely necessary so that the device recognizes the correct patient geometry for the body scan under consideration of the arms and can thus ensure an appropriate AEC.

The sex and age of the patient on the examination day (difference between birth and examination date) were recorded. Waist circumference was defined as the circumference of a region of interest (ROI) at skin level at the height of the navel. ( $\triangleright$ Fig. 5a). The injury severity was quantified via the ISS which was determined on the basis of the radiology $w b C T$ findings and the discharge letters [18]. The examination time was calculated as the difference between the parameters "study_time" (DICOM entry 0008|0030 - selection of the protocol at the CT unit) and the "acquisition_time" of the last image of the axial primary acquisition (DICOM entry $0008 \mid 0032)$. Thus, the scout acquisition, examination planning, contrast delay, actual scan time, and repositioning of the arms are taken into consideration. The objective image quality was determined as the standard deviation of the Hounsfield units in an ROI (= "image noise"). One ROI was placed in liver segment $\mathrm{VII}$ and one in the aorta ( $\triangleright$ Fig. 5b) $[19,20]$. The size and shape of the ROI were individually adapted to include as many voxels as possible and rule out vascular calcification, liver vessels, and parenchymal lesions (ROI circumference: Aorta $5.4 \pm 1 \mathrm{~cm}$; liver $9 \pm 1.6 \mathrm{~cm}$ ). From the dose reports in the PACS, the scan length (difference between start and end position) and the dose length product (DLP) were documented separately for the scans of the head/neck and body. The maximum CT dose index ( $\left.\mathrm{CTDI}_{\max }\right)$ documented at that time in the PACS dose reports is not suitable for a comparison to the literature. Therefore, the CTDI vol was calculated as the quotient of DLP and scan length. The effective dose (E) of the individual scans was calculated as the product of the relevant DLP and the corresponding conversion factor ( $\mathrm{k}$-factor). The $\mathrm{k}$-factors published by Huda for the head/neck scan $\left(k_{\text {Head } / \text { Neck }}=0.0045 \mathrm{mSv} / \mathrm{mGy}^{*} \mathrm{~cm}\right.$, in relation to $16 \mathrm{~cm}$ head phantom) and the body scan $\left(k_{\text {whole-Body }}=0.0154 \mathrm{mSv} / \mathrm{mGy}^{*} \mathrm{~cm}\right.$, in relation to $32 \mathrm{~cm}$ body phantom) according to ICRP103 [21, 22] were used for this purpose. $\mathrm{E}$ for a complete wbCT scan from vertex to ischium was calculated as the sum of the individual values.

The statistical analysis was performed with SPSS (Version 16.0, SPSS Inc., Chicago, IL, US). Extreme values (values outside the 3 rd interquartile range) were excluded from the analysis. The Kolmogorov-Smirnov test was used to test for normal distribution. Since there was no normal distribution in all three groups in any comparison, nonparametric tests were exclusively used. Therefore, results are specified as median and range (min-max). For better comparability with the literature, the mean and standard deviation (mean $\pm S D$ ) are additionally presented. The test for significance was performed with the Kruskal-Wallis test or Man$\mathrm{n}$-Whitney U-test. The level of significance was 0.05 .

\section{Results}

In the study period 308 patients fulfilled the inclusion and exclusion criteria (age: 46 a, $18-90$ a; $46 \pm 19$ a). Neither gender distribution (male: $\mathrm{n}=238 ;=77 \% ; \mathrm{P}=0.64$ ) nor waist circumference $(93 \mathrm{~cm}, 66-145 \mathrm{~cm} ; 94 \pm 15 \mathrm{~cm} ; \mathrm{P}=0.59)$ showed significant differences between the three groups.

The results regarding ISS and examination time and the ISS distribution within the groups are shown in $>$ Fig. $\mathbf{6 , 7}$. While the examination time varied only slightly between groups $\mathrm{O}$ and $\mathrm{T}$, the 


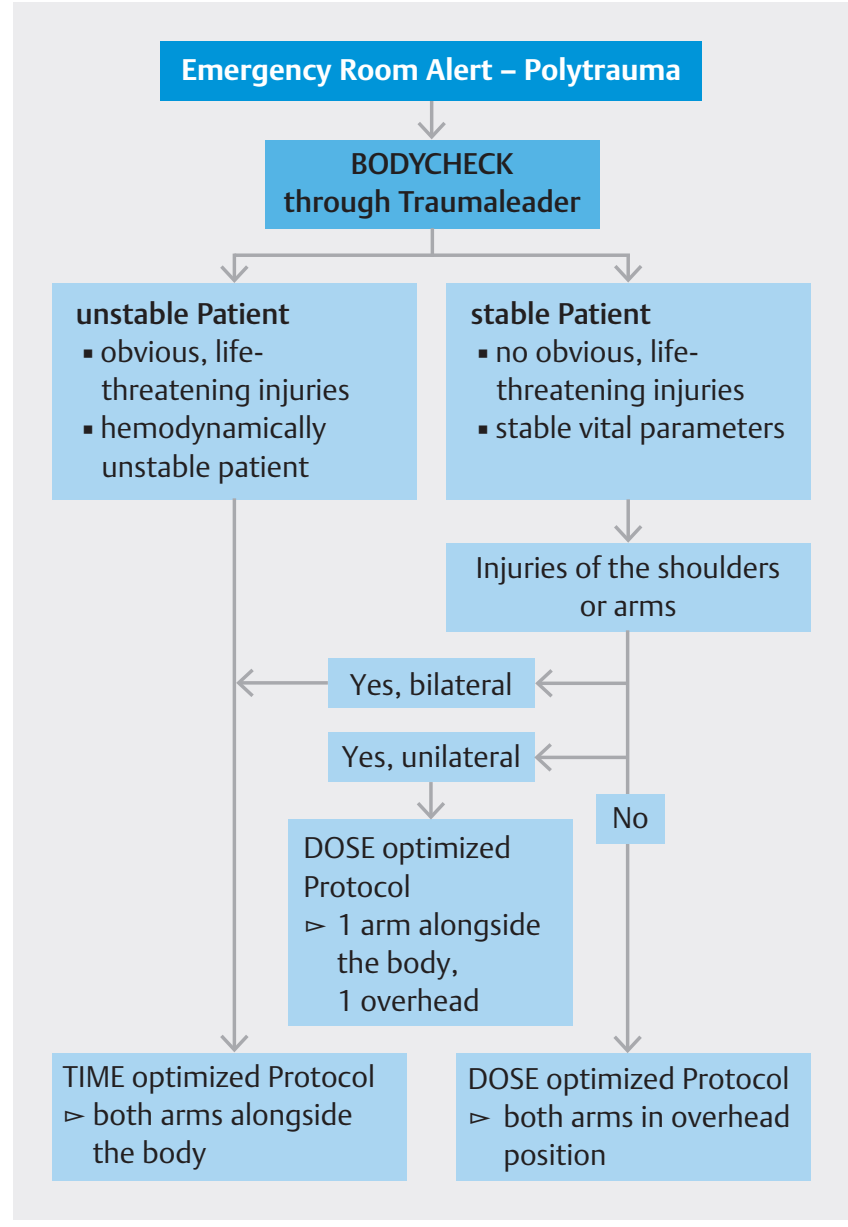

- Fig. 2 During emergency room treatment, the decision as to which CT protocol should be used (TIME optimized versus DOSE optimized Protocol) was made according to this scheme. In hemodynamically unstable patients the TIME protocol was used and in patients with stable vital parameters the DOSE protocol was used. If injuries of one or both arms were present in the initial body check, either the modified DOSE protocol with the injured arm alongside the body or the TIME protocol with both arms alongside the body was used.

examination time in group $\mathrm{D}$ was 3.6 minutes longer because the automatic examination procedure was not used.

The results regarding objective image quality are specified in - Fig. 8. There was no difference between groups $\mathrm{O}$ and $\mathrm{T}$, while significantly better image quality was seen in group $D$.

- Fig. 9 shows the determined dose values of the head/neck and body scans. There were no significant or clinically relevant differences in dose parameters between the three groups for the head/neck scans. In contrast, significant dose reductions could be achieved for the body scan. The comparison between groups $\mathrm{O}$ and T shows that the DLP is $37 \%$ lower $\left(927 \mathrm{mGy}^{*} \mathrm{~cm}\right)$ under optimized configuration of the AEC with the same image quality. In the comparison between groups $T$ and $D$, the DLP was able to be reduced by an additional $29 \%$ (466 $\left.\mathrm{mGy}^{*} \mathrm{~cm}\right)$ by repositioning the patient's arms.

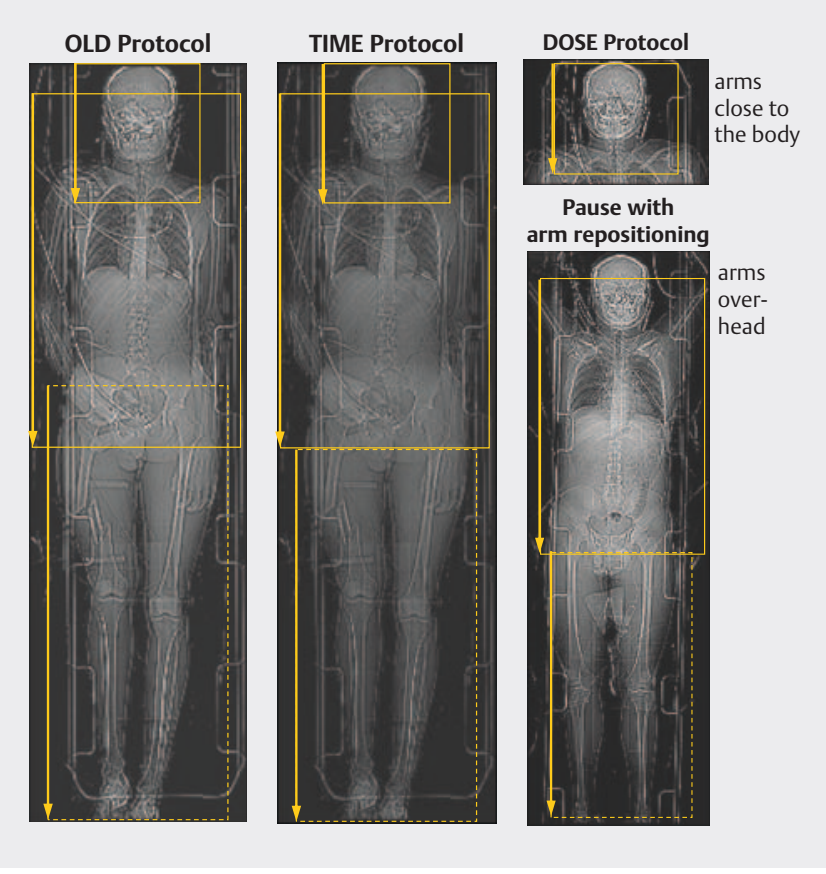

- Fig. 3 All of the three protocols evaluated in this study used the same scan sections: An unenhanced head/neck scan planned from vertex to thoracic vertebra 3 was followed by a contrast-enhanced scan of the body region planned from orbital roof to ischium. If severe injuries of the legs were suspected, a facultative subsequent scan of the legs was added. In the OLD protocol, the body and leg scans were planned with an overlap, which was explicitly avoided in both new protocols. The leg scans are not presented in this study. The OLD and TIME protocol had the same protocol design with arm positioning alongside the body and automated consecutively running scans. The DOSE protocol had a modified protocol design with a pause between the head/neck scan and body scan in which the arms were repositioned over the head. To ensure operational reliability of automatic exposure control, it is indispensable to acquire a new scout after repositioning the arms.

The average effective dose of a wbCT from vertex to ischium is compared in $>$ Fig. 10 to the dose reference values of the Federal Agency for Radiation Protection [23].

\section{Conclusion}

1. An optimized AEC setting can significantly reduce the radiation exposure even in the case of older devices.

2. Due to the constant further development of CT devices by manufacturers, existing protocols should be regularly reevaluated and optimized.

3. An examination of the body with the arms overhead has a longer examination time, but is superior with regard to image quality and dose to an examination with the arms positioned close to the body.

4. The clinically adapted dual concept with 2 differently weighted wbCT protocols is superior to the use of a single protocol for all patients ("one-fits-all concept"). 


\begin{tabular}{|c|c|c|c|}
\hline & OLD (O) & TIME (T) & DOSE (D) \\
\hline \multirow{2}{*}{ scouts } & \multicolumn{3}{|c|}{ acquisition of all scouts on 2 planes } \\
\hline & \multicolumn{3}{|c|}{ manual adjustment of scout length according to landmarks of requested scans ( $\rightarrow$ Fig. $\mathbf{3}$ ) } \\
\hline \multirow{5}{*}{ examination procedure } & \multirow{2}{*}{ 1. long whole-body scout } & \multirow{2}{*}{ 1. long whole-body scout } & 1. short head/neck scout \\
\hline & & & 2. head/neck Scan \\
\hline & $\begin{array}{l}\text { 2. simultaneus start of contrast } \\
\text { injector and CT-unit }\end{array}$ & \multirow{2}{*}{$\begin{array}{l}\text { 2. simultaneus start of contrast } \\
\text { injector and CT-unit }\end{array}$} & 3. pause with arm repositioning \\
\hline & & & 5. simult start of iniector /CT-unit \\
\hline & 3. automatic consecutive scans & 3. automatic consecutive scans & 6. automatic consecutive scans \\
\hline \multicolumn{4}{|c|}{ contrast agent $(100 \mathrm{ml}$ contrast agent $+20 \mathrm{ml} \mathrm{NaCl}$ with flow of $3 \mathrm{ml} / \mathrm{s})$} \\
\hline head/neck scan & native & native & native \\
\hline body scan & 60 s Delay & 60 s Delay & 60 s Delay \\
\hline leg scan & approx. 80 s Delay & approx. 80 s Delay & approx. 80 s Delay \\
\hline \multicolumn{4}{|c|}{ identical settings for all scans } \\
\hline tube voltage & $120 \mathrm{kV}$ & $120 \mathrm{kV}$ & $120 \mathrm{kV}$ \\
\hline rotation time & $0,5 \mathrm{~s}$ & $0,5 \mathrm{~s}$ & $0,5 \mathrm{~s}$ \\
\hline MPR creation & automatic & automatic & automatic \\
\hline MPR transfer to PACS & automatic & automatic & automatic \\
\hline \multicolumn{4}{|c|}{ head/neck scans (no changes made to the head/neck scans between protocols) } \\
\hline arms & close to the body & close to the body & close to the body \\
\hline tube current & $400 \mathrm{~mA}$ (AEC not active) & $400 \mathrm{~mA}$ (AEC not active) & $400 \mathrm{~mA}$ (AEC not active) \\
\hline convolution kernel & FC64 & FC64 & FC64 \\
\hline scan FOV & $320 \mathrm{~mm}$ & $320 \mathrm{~mm}$ & $320 \mathrm{~mm}$ \\
\hline collimation & $32 \times 0,5 \mathrm{~mm}$ & $32 \times 0,5 \mathrm{~mm}$ & $32 \times 0,5 \mathrm{~mm}$ \\
\hline pitch factor & 0,656 & 0,656 & 0,656 \\
\hline \multicolumn{4}{|l|}{ body scan } \\
\hline arms & close to the body & close to the body & overhead \\
\hline tube current & AEC active & AEC active & AEC active \\
\hline noise factor/SD value & $12 \mathrm{HU}$ & $14 \mathrm{HU}$ & $14 \mathrm{HU}$ \\
\hline AEC limits & $40-440 \mathrm{~mA}$ & $140-440 \mathrm{~mA}$ & $140-440 \mathrm{~mA}$ \\
\hline convolution kernel & FC15 & FC17 & FC17 \\
\hline scan FOV & $500 \mathrm{~mm}$ & $500 \mathrm{~mm}$ & $500 \mathrm{~mm}$ \\
\hline collimation & $32 \times 1 \mathrm{~mm}$ & $32 \times 1 \mathrm{~mm}$ & $32 \times 1 \mathrm{~mm}$ \\
\hline pitch factor & 0,844 & 0,844 & 0,844 \\
\hline
\end{tabular}

- Fig. 4 Examination protocols. Collimation: detector rows $\times$ slice thickness; MPR: multiplanar reconstructions; AEC: automatic exposure control;

FOV: field-of-view.

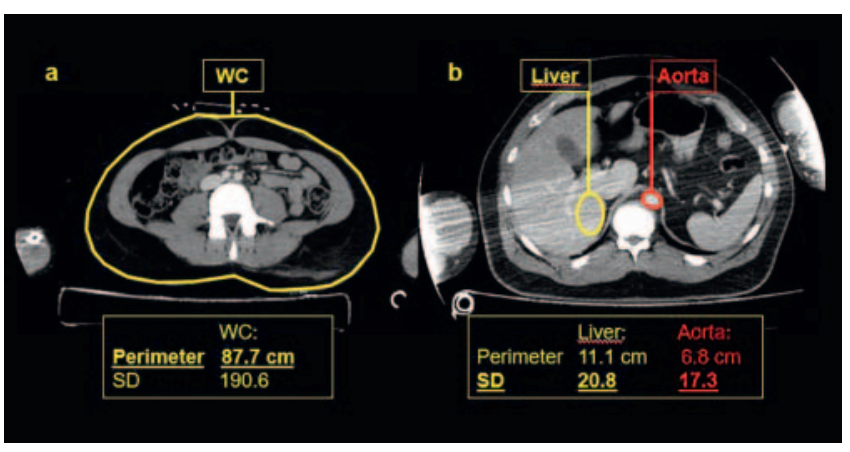

- Fig. 5 a Waist circumference was defined as the circumference of a freehand ROI at skin level at the height of the navel. $\mathbf{b}$ Objective image quality was defined as "image noise" (= standard deviation of Hounsfield units in an ROI). The ROIs were placed in liver segment VII and in the aorta.
5. WbCT scans result in relevant radiation exposure. Multicenter studies quantifying the average radiation exposure of a wbCT scan are needed.

\section{Discussion}

In the comparison between groups $\mathrm{O}$ and $\mathrm{T}$, optimizing the $\mathrm{AEC}$ results in a reduction in radiation exposure with a similar examination time and stable image quality. At an AEC setting identical to that of group $\mathrm{T}$, the repositioning of the patient's arms and the modified protocol design in group $D$ result in a longer examination time with reduced radiation exposure and improved image quality.

The patient group examined in the present study is to be considered representative compared to the TraumaRegister (annual report 2016: Mean $_{\text {Age }} 51.4$ a; $70 \%$ male) [6].

Since CT takes the patient's habitus into consideration based on scouts when using AEC, it has a direct influence on dose. Since there were no significant differences regarding waist circumfer- 


\begin{tabular}{|c|c|c|c|c|c|c|c|c|c|}
\hline & \multicolumn{2}{|c|}{ OLD (0) } & \multicolumn{2}{|c|}{ TIME (T) } & \multicolumn{2}{|c|}{ DOSE (D) } & \multicolumn{3}{|c|}{ P-Values } \\
\hline & Median & Range & Median & Range & Median & Range & \multirow{2}{*}{ O - T } & \multirow{2}{*}{ O - D } & \multirow{2}{*}{$T-D$} \\
\hline & $\mathrm{MW}$ & $\pm S D$ & $\mathrm{MW}$ & $\pm S D$ & MW & $\pm S D$ & & & \\
\hline \multirow{2}{*}{$\begin{array}{c}\text { ET } \\
\text { in } \min \end{array}$} & $3,93^{(*)}$ & $3,3-5,63$ & $4,1^{(*)}$ & $2,8-7,17$ & $7,72^{(*)}$ & $6-10$ & $\mathrm{~s}$ & $\mathrm{~s}$ & $\mathrm{~s}$ \\
\hline & $3,98^{(*)}$ & $\pm 0,45$ & $4,27^{(*)}$ & $\pm 0,83$ & $7,73^{(*)}$ & $\pm 0,82$ & & & \\
\hline \multirow{2}{*}{ ISS } & $14^{(*)}$ & $0-75$ & $18^{(*)}$ & $0-75$ & $9^{(*)}$ & $0-50$ & $\mathrm{~s}$ & $\mathrm{~s}$ & $\mathrm{~s}$ \\
\hline & $17,2^{(*)}$ & $\pm 16,5$ & $21,3^{(*)}$ & $\pm 16,1$ & $10,7^{(*)}$ & $\pm 10,5$ & & & \\
\hline
\end{tabular}

- Fig. 6 This figure presents the results of examination time and ISS as median and range. For comparison mean value and standard deviation are given additionally. $\mathrm{s}=$ significant; $\mathrm{ns}=$ not significant.

${ }^{*}$ In the analysis of examination time, some extreme values (values out of $3 \mathrm{rd} \mathrm{IQR)} \mathrm{were} \mathrm{excluded} \mathrm{from} \mathrm{further} \mathrm{analysis} \mathrm{(group} \mathrm{OLD} \mathrm{(O):} 3$, group TIME (T): 3, group DOSE (D): 2).

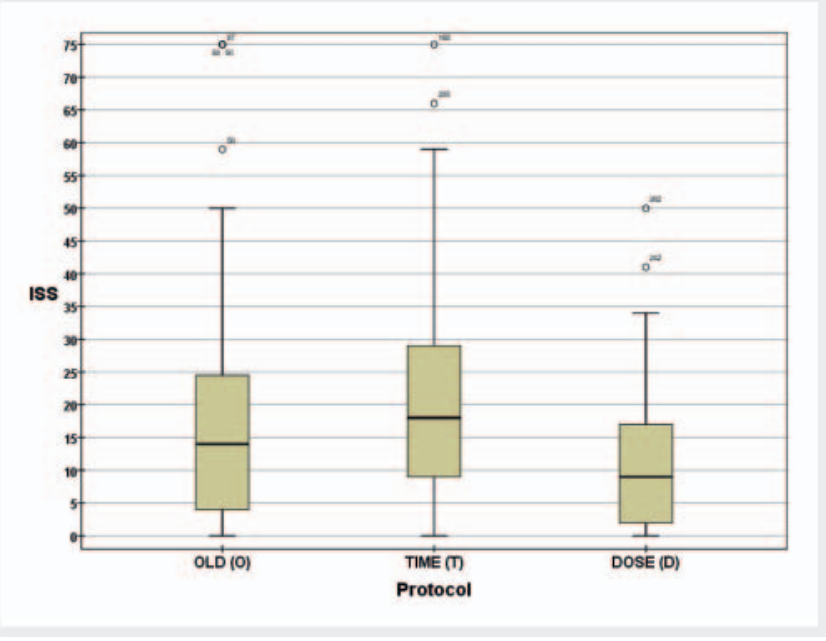

Fig. 7 The ISS was calculated using the findings of the wholebody CT scan and diagnoses from the hospital discharge letter [18]. The OLD protocol was used for all patients in the sense of a "onefits-all" concept. Since 2011, two new and differently weighted CT protocols have been implemented ( $\vee$ Fig. 2 ). From this data it is evident that more severely injured patients were found in the TIME group, and more minorly injured patients in the DOSE group. This reflects the triage carried out in the emergency room.

The box represents the interquartile range (IQR) which contains the middle $50 \%$ of the ISS values. The line across the box indicates the median. The whiskers (lines that extend from the upper and lower edge of the box) represent the highest and lowest values which are no greater than 1.5 times the IQR. Outliers are values beyond the whiskers between 1.5 and 3 times the IQR.

ence between the three groups, there was no falsification of dose values due to asymmetrical distribution of patients with a different habitus among the groups.

Comparison of the three groups showed a clear injury severity dynamic ( $\triangleright$ Fig. 7). In the OLD protocol previously used for all patients, the ISS covers the complete range of $0-75$ points. In contrast, higher ISSs have been redistributed to group T and lower scores to group D since implementation of the clinically adapted procedure. This reflects the clinical triage done in our emergency room. In total, the ISS was higher than 15 points in 139 patients, theoretically resulting in overtriage of $54.9 \%$ of patients according to Wurmb [17]. The literature shows a broad range of published ISS values: Huber-Wagner: ISS mean $28.8 \pm 12.1$ [2], Yaniv: mean $13 \pm 11.2$ [24] or median 5 (IQR 1 - 13) [16]. Differences can be primarily explained by variations in study design. Many studies on polytrauma wbCT neglect this important characteristic of trauma patients. Therefore, future studies should additionally determine the more precise NISS (new ISS).

With the same protocol design, there was a similar examination time in groups $\mathrm{O}$ and $\mathrm{T}$ (approx. $4 \mathrm{~min}$ ). As a result of the modified protocol design, the examination time in group $D$ increased to 7.7 minutes. A literature comparison is only possible on a limited basis since the definitions of examination time vary. The scan time (= time that radiation is emitted) is unsuitable as a reference value (e. g. 35 - 65 s [25]). The definition of examination time used in this study, i. e., the difference between protocol selection at the CT device and acquisition time of the last axial image of the primary acquisition, takes into account scout acquisition, planning, scan time, contrast delay, and any arm repositioning and is thus a realistic variable. Extreme values (life-saving procedures, reanimation) were excluded from the statistical analysis. The optimal definition of examination time to be preferred by future authors was used by Yaniv and Bayer (difference between acquisition time of the first scout image and the last axial image) $[24,26]$. The examination time of $14.1 \pm 4.1$ to $14.3 \pm 9.5$ minutes or $7: 30 \pm 2: 52 \mathrm{~min}: \mathrm{s}$ is comparable with the present study. In the case of unstable patients requiring a short wbCT examination time, the protocol design of groups $\mathrm{O}$ and $\mathrm{T}$ with an examination time of 4 minutes is a real time-optimized protocol.

The occurrence of typical artifacts with the patient's arms close to the body is generally known and is common in obese patients ( $\triangleright$ Fig. 11). The definition of objective image quality used in this study provides values for organ-specific image noise and is used to compare the protocols evaluated here. In the case of differences regarding CT unit, AEC functionality, and ROI position, comparison of the individual values with other studies is not useful. Other studies with a similar method come to the same conclusion: wbCT scans with the patient's arms close to the body have worse image quality and a higher dose than scans with the patient's arms overhead [19, 20].

Since the head/neck scans were examined with the same setting, no differences could be identified between the groups as expected. It must be noted here that the neck was overexposed when examining this region with a constant tube current and a dose for intracranial image quality. This is reflected in the dose values that exceed the dose reference values of the Federal Agen- 


\begin{tabular}{|c|c|c|c|c|c|c|c|c|c|}
\hline & \multicolumn{2}{|c|}{ OLD (O) } & \multicolumn{2}{|c|}{ TIME (T) } & \multicolumn{2}{|c|}{ DOSE (D) } & \multicolumn{3}{|c|}{ P-values } \\
\hline & Median & Range & Median & Range & Median & Range & \multirow{2}{*}{$O-T$} & \multirow{2}{*}{$O-D$} & \multirow{2}{*}{$T-D$} \\
\hline & $\mathrm{MW}$ & $\pm S D$ & $\mathrm{MW}$ & $\pm S D$ & $\mathrm{MW}$ & $\pm S D$ & & & \\
\hline \multirow{2}{*}{$\begin{array}{l}\text { IN Aorta } \\
\text { (in HU) }\end{array}$} & 17 & $11,1-39,5$ & $16^{(*)}$ & $10,5-35,3$ & 14 & $9,7-19,9$ & ns & $s$ & $\mathrm{~s}$ \\
\hline & 18,6 & $\pm 5,3$ & $17,3^{(*)}$ & $\pm 4,8$ & 14,1 & $\pm 2,2$ & & & \\
\hline \multirow{2}{*}{$\begin{array}{c}\text { IN Leber } \\
\text { (in HU) }\end{array}$} & 19,4 & $7,5-56,3$ & $18,3^{(*)}$ & $11,3-30,4$ & 14,7 & $10,9-18,9$ & ns & $\mathrm{s}$ & $\mathrm{s}$ \\
\hline & 21 & $\pm 6,9$ & $19,2^{(*)}$ & $\pm 4,1$ & 14,6 & $\pm 1,8$ & & & \\
\hline
\end{tabular}

- Fig. 8 The objective image quality was defined as image noise (=IN = standard deviation of Hounsfield units in a ROI). The respective ROIs were placed in one slice at the level of the upper abdomen in liver segment VII and in the aorta. In addition to the results (Median/Range) mean value and standard deviation are presented for better comparison to literature. $s=$ significant; $n s=$ not significant.

* In the analysis of image noise, some extreme values (values outside of the $3 \mathrm{rd}$ IQR) were excluded from further analysis (group TIME (T): aorta-2, liver-4).

\begin{tabular}{|c|c|c|c|c|c|c|c|c|c|c|c|}
\hline & \multirow{2}{*}{\multicolumn{2}{|c|}{ head/neck scan }} & \multicolumn{6}{|c|}{ body scan } & \multirow{2}{*}{\multicolumn{3}{|c|}{$P$ - values }} \\
\hline & & & \multicolumn{2}{|c|}{ OLD (0) } & \multicolumn{2}{|c|}{ TIME (T) } & \multicolumn{2}{|c|}{ DOSE (D) } & & & \\
\hline & Median & Range & Median & Range & Median & Range & Median & Range & $0 \mathrm{~T}$ & ח & T \\
\hline & MW & $\pm S D$ & $\mathrm{MW}$ & $\pm S D$ & $\mathrm{MW}$ & $\pm S D$ & $\mathrm{MW}$ & $\pm S D$ & $0-1$ & $0-D$ & $1-D$ \\
\hline \multirow{2}{*}{$\begin{array}{l}\text { scan length } \\
(\mathrm{cm})\end{array}$} & 34,7 & $29,2-42,1$ & 93,9 & $80,9-107,9$ & 91,9 & $78,9-108,4$ & 91,9 & $81,9-106,4$ & $\mathrm{~s}$ & $\mathrm{~s}$ & ns \\
\hline & 35 & $\pm 1,7$ & 94,2 & $\pm 5,3$ & 91,8 & $\pm 5,3$ & 91,6 & $\pm 5,4$ & & & \\
\hline \multirow{2}{*}{$\begin{array}{l}\mathrm{CTDI}_{\text {vol }} \\
(\mathrm{mGy})\end{array}$} & 70,4 & $70,3-70,6$ & 26,9 & $17,2-30,2$ & 17 & $10,4-28,2$ & 12,1 & $10,4-29,6$ & $\mathrm{~s}$ & $\mathrm{~s}$ & $\mathrm{~s}$ \\
\hline & 70,4 & $\pm 0,1$ & 26,3 & $\pm 2,3$ & 17,8 & $\pm 5,4$ & 14,8 & $\pm 5,3$ & & & \\
\hline \multirow{2}{*}{$\begin{array}{c}\mathrm{DLP} \\
\left(\mathrm{mGy}{ }^{*} \mathrm{~cm}\right)\end{array}$} & 2437 & $2054-2965$ & 2512 & $1388-2969$ & 1585 & $892-2679$ & 1119 & $859-3030$ & $\mathrm{~s}$ & $\mathrm{~s}$ & $\bar{s}$ \\
\hline & 2464 & \pm 123 & 2481 & \pm 268 & 1633 & \pm 520 & 1360 & \pm 513 & & & \\
\hline \multirow{2}{*}{$E(m S v)$} & 11 & $9,2-13,3$ & 38,7 & $21,4-45,7$ & 24,4 & $13,7-41,3$ & 17,2 & $13,2-46,7$ & $\mathrm{~s}$ & $\mathrm{~s}$ & $\bar{s}$ \\
\hline & 11,1 & $\pm 0,6$ & 38,2 & $\pm 4,1$ & 25,2 & \pm 8 & 21 & $\pm 7,9$ & & & \\
\hline
\end{tabular}

- Fig. 9 This figure presents the dose evaluations of the single scans as median and range. Mean values and standard deviation are given for better comparison to literature additionally. Scan length and dose length product (DLP) incl. overranging. The effective dose E was calculated using conversion factors according to the ICRP103 [21, 22]. Because of identical settings in the head/neck scans, no differences were found among the three groups (reference to the $16 \mathrm{~cm}$ head phantom). In contrast, through optimization of automatic exposure control (comparing group OLD with TIME) and through repositioning of the arms (comparing group TIME with DOSE), significant dose reductions were achieved in the body scan (reference to the $32 \mathrm{~cm}$ body phantom). $\mathrm{s}=$ significant; $\mathrm{ns}=$ not significant

\begin{tabular}{|c|c|c|c|c|c|c|c|c|}
\hline section 1 & OLD (0) & TIME (T) & DOSE (D) & section 2 & $\begin{array}{l}\text { DLP-DRV old } \\
\text { (in } m G y^{*} \mathrm{~cm} \text { ) }\end{array}$ & $\begin{array}{c}\mathrm{DRV}_{\text {old }}{ }^{-} \\
\mathrm{E}_{\text {Huda }} \text { (in mSv) }\end{array}$ & $\begin{array}{l}\text { DLP-DRV } \\
\text { (in } \mathrm{mGy}^{*} \mathrm{~cm} \text { ) }\end{array}$ & $\begin{array}{c}\mathrm{DRV}_{\text {new }}{ }^{-} \\
\mathrm{E}_{\text {Huda }} \text { (in } \mathrm{mSv} \text { ) }\end{array}$ \\
\hline \multirow{2}{*}{ CCT/neck CT } & \multirow{2}{*}{$11 \mathrm{mSv}$} & \multirow{2}{*}{$11 \mathrm{mSv}$} & \multirow{2}{*}{$11 \mathrm{mSv}$} & СCT & 950 & 2,28 & 850 & 2,04 \\
\hline & & & & Carotid angio & $(600)$ & $(6,42)$ & 600 & 6,42 \\
\hline \multirow{3}{*}{ neck and trunk $\mathrm{CT}$} & \multirow{3}{*}{$38,7 \mathrm{mSv}$} & \multirow{3}{*}{$24,4 \mathrm{mSv}$} & \multirow{3}{*}{$17,2 \mathrm{mSv}$} & Thorax & 400 & 8,16 & 350 & 7,14 \\
\hline & & & & Upper abdomen & 450 & 7,34 & 360 & 5,87 \\
\hline & & & & Pelvis (soft tissue) & 450 & 6,44 & 400 & 5,72 \\
\hline total & $\underline{49,7 \mathrm{mSv}}$ & $\underline{35,4 \mathrm{mSv}}$ & $\underline{28,2 \mathrm{mSv}}$ & & & $(30,64 \mathrm{mSv})$ & & $\underline{27,19 \mathrm{mSv}}$ \\
\hline
\end{tabular}

- Fig. 10 In section 1 for each protocol the effective doses of the single scan regions ( $\triangleright$ Abb. 9) were added to calculate the mean effective dose for a whole-body CT scan from vertex to ischium. For reference in section 2, the dose reference values (= DRVs) of the German Federal Office for Radiation Protection are shown in column DLP-DRV old $_{\text {and column DLP-DRV }}$ (updated in 2016). There was no value in the old DRV for the CT scan of the neck region. The corresponding value of the new DRV was used instead. The DRVs were multiplied with conversion factors according to ICRP103 $[21,22]$ to calculate "effective dose reference values", shown in column DRV ${ }_{\text {old }}-E_{\text {Huda }}$ and DRV $V_{\text {new }}-E_{\text {Huda }}$. The aim was to generate a maximum limit for an elective whole-body CT scan. All effective doses found in this study exceeded the current DRVs. When using whole-body CT as an emergency diagnostic modality in multiple trauma, it is, however, acceptable to exceed the DRV.

cy for Radiation Protection ( $\triangleright$ Fig. 9, 10) [23]. This aspect was identified as an object of future protocol optimization.

Comparing body scans shows similar scan lengths between groups $T$ and $D$ which are shorter than those in group $O$. This is a reasonable result of the increased planning by technologists in the new protocols since the overlapping of body and leg scans is to be explicitly avoided.
In the group comparison of dose values, significant dose reductions ( $>$ Fig. 9) were achieved both by optimizing the AEC using current software (O-T) and by repositioning the patient's arms (T-D). Both aspects are considered relevant variables influencing dose and image quality [19, 20, 27, 28]. In the case of an identical AEC setting, the difference in the positioning of the arms between groups $T$ and $D$ is the only possible explanation for the variations in image quality and dose ( $\triangleright$ Fig. 4 ). The dose reduction 


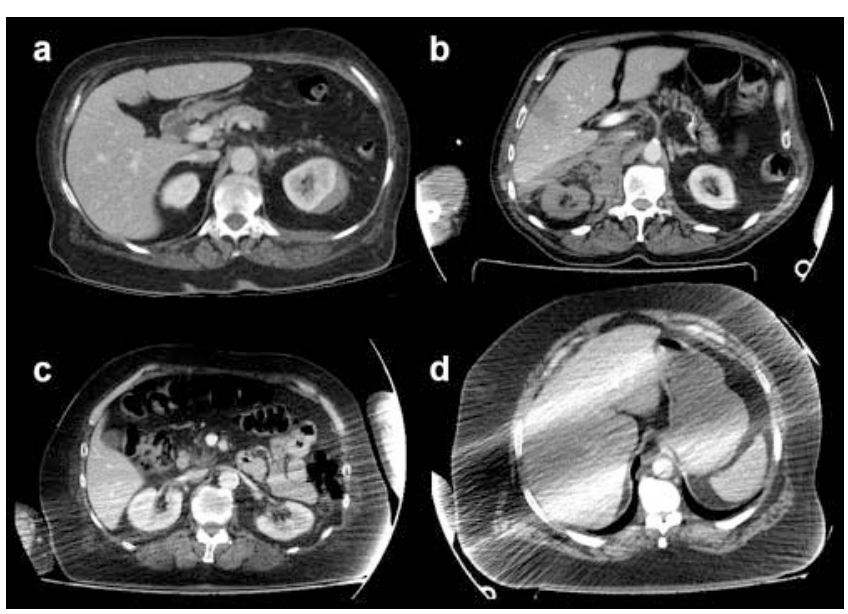

- Fig. 11 The figure demonstrates different examples of single slices of body scans with arms over the head (group DOSE - ex. a) and arms close to body (group TIME - ex. $\mathbf{b}$ - d). Characteristic beamhardening artifacts due to the positioning of the arms are present and can significantly impact the interpretation of the diagnostic procedure, especially in obese patients.

resulting from the repositioning of the patient's arms was $7 \mathrm{mSv}$ according to Brink which is comparable with the present study (7.2 mSv) [20]. However, such values cannot be generalized since significant differences can occur when examining the same patient on a device by the same manufacturer simply due to the use of a different AEC setting. It can thus be concluded that existing protocols must be regularly reevaluated and optimized due to the constant further developments made by $\mathrm{CT}$ device manufacturers.

The dose reference values of the Federal Agency for Radiation Protection were updated in 2016 and provide guidelines to be observed during typical examinations of standard patients (body weight $70 \pm 3 \mathrm{~kg}$ ) [23]. Depending on the indication, habitus, and special aspects (metal implants) and particularly in the case of emergency diagnostic imaging, it is acceptable to exceed the dose reference values. $>$ Fig. 10 shows that the doses particularly in groups $\mathrm{O}$ and $\mathrm{T}$ were above the current dose reference values, with the doses published here being acquired in the validity period of the old dose reference values between 2011 and 2013. Additional protocol optimizations have since been performed and are the subject of a planned follow-up study.

The general opinion in the literature is that wbCT from vertex to ischium corresponds to approx. $10-20 \mathrm{mSv}$ [13]. The present values as well as the speculation of Ruchholtz (30.05 mSv) contradict this [9]. Harrieder describes a broad range of dose values for $\mathrm{wbCT}$ in the literature [29]. From our point of view there are many reasons for this:

1. There are various wbCT protocols partially with multiphase scans and multiple overlaps.

2. Many studies publish only dose values for body scans neglecting the head/neck scans.

3. Some studies provide data regarding radiation exposure [19, $20,24,27,29]$, while others use phantom studies and others provide estimated values that cannot be reproduced.
4. Dose values are published in a highly varied and sometimes incomplete manner. In addition, different k-factors based on either tissue weighting factors according to ICRP 60 or the current ICRP 103 are used [21, 22, 30]. Depending on the published, dose-relevant parameters, conversion and thus comparability with other studies can be limited or even impossible. Converting the published values using the k-factors from Huda to values comparable with this study would result in dose values of 41.1 - 49.5 mSv [25], 26.2 - $28 \mathrm{mSv}$ [29], 15.7 $20.2 \mathrm{mSv}$ [27], $11.4-28.2 \mathrm{mSv}$ [20] or $12.4-18.2 \mathrm{mSv}$ [24]. It must be explicitly noted here that the last three studies provided values only for body scans but not for head/neck scans. To date, no anonymized retrospective multicenter evaluation of this topic has been performed.

A comparison of a "one-fits-all" concept with the clinically adapted concept using the latest hardware and software yields the following points:

Supplementing the prior exclusive use of a protocol with the patient's arms close to the body for all patients (TIME protocol) with the addition of a DOSE protocol makes it possible to examine stable patients according to the ALARA principle with an adequate dose and better image quality. This results in reduced collective radiation exposure which particularly benefits slightly injured patients who were overtriaged in the emergency room.

In the case of the previous sole use of a DOSE protocol with repositioning of the patient's arms overhead for all patients, particularly severely injured, hemodynamically unstable patients benefit from the addition of a TIME protocol due to the reduced examination time and the more time-efficient care. Higher radiation exposure is acceptable for these patients during emergency diagnostic imaging.

By using differently weighted wbCT protocols, each patient's clinical situation can be approached with greater flexibility on an individualized basis.

In patients with a relevant mechanism of trauma, there is an alternative diagnostic concept consisting of the combination of ultrasound, X-rays of the skeleton of the trunk and if necessary subsequent CT of individual body regions. Adding up the literature values yields an effective dose of $3.32 \mathrm{mSv}$ for X-ray imaging of the cervical spine, thoracic spine, lumbar spine, thorax, and pelvis [31]. This corresponds to $1 / 10$ the dose of one wbCT ( $\triangleright$ Fig. 10). Imaging of the thorax p.a. corresponds to $0.02 \mathrm{mSv}$. Therefore, one wbCT at $30 \mathrm{mSv}$ would equal 1500 chest X-rays.

According to the Federal Agency for Radiation Protection, the average annual radiation exposure in Germany from natural and civilization-based sources is $4 \mathrm{mSv}$. Trauma patients are often very young, and they have often only encountered natural radiation exposure $(2.1 \mathrm{mSv})$. wbCT with $30 \mathrm{mSv}$ would therefore correspond to natural radiation exposure of $14.3 \mathrm{a}$.

\section{Limitations}

The basic limitations of this study are its retrospective, monocentric, unblinded, and non-randomized design. 
Particularly in obese patients, there is high variability regarding the position of the navel. Therefore, future studies should determine the waist circumference along the $X$-axis not based on the navel as in this study but based on bony landmarks (e. g. L3).

In the case of examinations with the arms close to the body, the actual body volume exposed to radiation is represented incompletely through the waist circumference. Therefore, no correlations between waist circumference and dose are presented in this study.

The definition of examination time that is used in this study is susceptible to outliers, e. g. due to prolonged emergency room care. The definition used by Yaniv and Bayer is to be given preference for future studies $[24,26]$.

In the analysis of examination time, no differentiation was made between wbCT with and without a leg scan. This results in inaccuracies in the examination time in patients who underwent a leg scan (frequency: T>O > D) of approx. 20 s (5 s interscan delay with respect to the body scan and a scan time of approx. $15 \mathrm{~s}$ for leg scans). This explains the difference in examination time between groups $\mathrm{O}$ and $\mathrm{T}$ in particular.

The examination of all patients on a CT-compatible spine board is a further limitation as it can have a significant effect on dose and image quality [32]. This was not evaluated in this study. Since all patients were examined in this way, this represents a systematic error and is to be ignored within this study.

\section{Conclusion}

Acute care of polytraumas is an interdisciplinary challenge for trauma centers. There are usually interdisciplinary guidelines for procedures that can differ significantly between various hospitals. Diagnosis via wbCT is an important component of a total concept and is subject to complex influences and interactions. In addition to technical factors of the CT unit (manufacturer, product, software options, maximum scan length, iterative reconstructions) and the actual device settings (tube voltage, rotation time, $A E C$ ), spatial, logistic, and interdisciplinary aspects (CT in the emergency room or separate rooms, emergency room care procedures, head/feet-first position, arm position, contrast regime) also affect the protocol design. Due to this high complexity and based on the focus compromises must always be made compared to elective standard protocols for individual body regions. There are many publications addressing protocol optimization in wbCT, for example in relation to the manufacturer, in relation to the device, in relation to individual aspects of protocol design, and in relation different designs compared to one another. A multicenter study more precisely quantifying the average radiation exposure caused by wbCT is not yet available.

Additional research regarding the topic of indication determination is also needed to develop a practical concept that rules out undertriage to the greatest extent possible and keeps the overtriage rate relatively low. The present study describes a clinically adapted concept that can be integrated between indication determination and the performance of wbCT. It allows a more flexible approach to individual aspects of trauma patients in the emergency room and provides an option for lowering the collective radiation exposure even when performing wbCT.

\section{CLINICAL RELEVANCE OF THE STUDY}

- The present study describes a concept for wbCT in patients with multiple injuries: The use of 2 differently weighted protocols allows a more flexible approach to the clinical presentation of trauma patients in the emergency room.

- The time-optimized wbCT protocol targets severely injured patients. The focus is on the shortest possible examination time with compromises being made regarding image quality and radiation exposure.

- The dose-optimized wbCT protocol targets more slightly injured, possibly overtriaged patients. The focus is on the lowest possible radiation exposure with the use of as many dose reduction options as possible.

- The clinically adapted concept presented here may be a further step in improving the acute care of trauma patients in Germany with the goal of reducing the collective dose.

\section{Conflict of Interest}

The authors declare that they have no conflict of interest.

\section{Acknowledgments}

The present study was performed between 2010 and 2015 (data acquisition 2010 - 2013) at BG Hospital Bergmannstrost in Halle (Saale). It is the publication of an excerpt from the doctoral thesis of the first author. We would like to thank the Department of Diagnostic Imaging and Interventional Radiology (BG-Klinikum Bergmannstrost, Halle (Saale), Germany) for providing the data that was used.

\section{References}

[1] Statistisches Bundesamt. Gesundheit - Todesursachen in Deutschland 2013. 2014 https://www.destatis.de/DE/Publikationen/Thematisch/ Gesundheit/Todesursachen/Todesursachen.html Abgerufen am: 03.12.2014

[2] Huber-Wagner S, Lefering R, Qvick LM, Working Group on Polytrauma of the German Trauma Society et al. Effect of whole-body CT during trauma resuscitation on survival: a retrospective, multicentre study. Lancet 2009; 373: $1455-1461$

[3] Salim A, Sangthong B, Martin M et al. Whole body imaging in blunt multisystem trauma patients without obvious signs of injury: Results of a prospective study. Arch Surg 2006; 141: 468-475

[4] Hoffstetter P, Herold T, Daneschnejad M et al. Non-trauma-associated additional findings in whole-body CT examinations in patients with multiple trauma. Fortschr Röntgenstr 2008; 180: 120-126

[5] TraumaRegister DGU® Jahresbericht 2009. 2009 http://www.traumaregister-dgu.de/de/service/downloads.html Abgerufen am: 15.11.2017

[6] TraumaRegister DGU® Jahresbericht 2017. 2017 http://www.traumaregister-dgu.de/de/service/downloads.html Abgerufen am: 15.11.2017

[7] Snyder GE. Whole-body imaging in blunt multisystem trauma patients who were never examined. Ann Emerg Med 2008; 52: 101 - 103 
[8] Fabian TC. Whole-body CT in multiple trauma. Lancet 2009; 373: 1408 1409

[9] Ruchholtz S, Waydhas C, Schroeder T et al. The value of computed tomography in the early treatment of seriously injured patients. Chirurg 2002; 73: $1005-1012$

[10] Brenner DJ, Hall EJ. Cancer Risks from CT Scans: Now We Have Data, What Next? Radiology 2012; 265: 330-331

[11] Laack TA, Thompson KM, Kofler JM et al. Comparison of trauma mortality and estimated cancer mortality from computed tomography during initial evaluation of intermediate-risk trauma patients. J Trauma 2011; 70: $1362-1365$

[12] AUC TraumaRegister DGU ®. http://www.traumaregister-dgu.de/de/registerstruktur.html Abgerufen am 15.11.2017

[13] Deutsche Gesellschaft für Unfallchirurgie. S3-Leitlinie Polytrauma/ Schwerverletzten-Behandlung. 2016 http://www.awmf.org/leitlinien/ detail/I/012-019/II-ansicht/autor.html Abgerufen am: 15.11.2017

[14] Kloth JK, Kauczor HU, Hosch W. Imaging in the emergency room. Med Klin Intensivmed Notfmed 2011; 106: 82 - 88

[15] Huber-Wagner S et al. Whole-body CT Score - Kriterien zur Durchführung einer Ganzkörper-Computertomografie bei potentiell schwerverletzten Patienten. Berlin: Deutscher Kongress für Orthopädie und Unfallchirurgie (DKOU 2015). 2015

[16] Gupta M, Schriger DL, Hiatt JR et al. Selective use of computed tomography compared with routine whole body imaging in patients with blunt trauma. Ann Emerg Med 2011; 58: 407 -416.e15

[17] Wurmb TE, Kenn W. The role of early multislice computed tomography in major trauma. Trauma 2012; 14: $301-312$

[18] Baker SP, O’Neill B. The injury severity score: an update. J Trauma 1976; 16: $882-885$

[19] Karlo C, Gnannt R, Frauenfelder T et al. Whole-body CT in polytrauma patients: effect of arm positioning on thoracic and abdominal image quality. Emerg Radiol 2011; 18: 285-293

[20] Brink M, de Lange F, Oostveen L] et al. Arm Raising at Exposure-controlled Multidetector Trauma CT of Thoracoabdominal Region: Higher Image Quality, Lower Radiation Dose. Radiology 2008; 249: 661 - 670
[21] Huda W, Magill D, He W. CT effective dose per dose length product using ICRP 103 weighting factors. Med Phys 2011; 38: 1261-1265

[22] International Commission on Radiological Protection. The 2007 Recommendations of the ICRP. ICRP Publication 103. Ann ICRP. 2007; 37: (2-4).

[23] Bundesamt für Strahlenschutz Deutschland. Diagnostische Referenzwerte für diagnostische und interventionelle Röntgenanwendungen. 2016 http://www.bfs.de/DE/themen/ion/anwendung-medizin/diagnostik/referenzwerte/referenzwerte_node.html Abgerufen am: 15.11.2017

[24] Yaniv G, Portnoy O, Simon D et al. Revised protocol for whole-body CT for multi-trauma patients applying triphasic injection followed by a single-pass scan on a 64-MDCT. Clin Radiol 2013; 68: 668-675

[25] Fanucci E, Fiaschetti V, Rotili A et al. Whole body 16-row multislice CT in emergency room: effects of different protocols on scanning time, image quality and radiation exposure. Emerg Radiol 2007; 13: 251 - 257

[26] Bayer J, Pache G, Strohm PC et al. Influence of arm positioning on radiation dose for whole body computed tomography in trauma patients. J Trauma 2011; 70: $900-905$

[27] Loewenhardt B, Buhl M, Gries A et al. Radiation exposure in whole-body computed tomography of multiple trauma patients: bearing devices and patient positioning. Injury 2012; 43: $67-72$

[28] Kalender WA, Buchenau S, Deak P et al. Technical approaches to the optimisation of CT. Phys Med 2008; 24: 71 - 79

[29] Harrieder A, Geyer LL, Körner M et al. Evaluation of radiation dose in 64 row whole-body CT of multiple injured patients compared to 4-row CT. Fortschr Röntgenstr 2012; 184: 443 -449

[30] International Commission on Radiological Protection. 1990 Recommendations of the ICRP. ICRP Publication 60. Ann ICRP. 1991; 21: (1-3)

[31] Mettler FA, Huda W, Yoshizumi TT et al. Effective Doses in Radiology and Diagnostic Nucelar Medicine: A Catalog. Radiology 2008; 248: 254 - 263

[32] Loewenhardt B, Huettinger R, Reinert $M$ et al. Dose effects and image quality: Is there any influence by bearing devices in whole-body computed tomography in trauma patients? Injury 2014; 45: 170-175 